\title{
La cosa que se traza a sí misma: límites y aporías del aparecer sensible desde la fenomenología temprana*
}

\author{
Felipe Johnson \\ Universidad de La Frontera, Chile \\ felipe.johnson@ufrontera.cl \\ https://orcid.org/0000-0001-6063-9322
}

Resumen: Este artículo se propone indagar la presencia de las cosas sensibles a la luz de los trabajos de la fenomenología temprana. Para ello se elaborará una reflexión desde las problemáticas que algunos pensadores del círculo de Gotinga esbozaron en el contexto de las tesis husserlianas referentes a la Abschattung. Así, distinguiremos entre las cosas y su trazo sensible, detectando las posibilidades de su mutua vinculación y desvinculación fenoménica. Por esta vía, se identificará una relación triple en la que la cosa empírica aparece, compuesta por las cualidades sensibles, las sensaciones del sujeto percipiente y su ubicación espacial, para examinar cuál de tales instancias explica el necesario vínculo entre trazo sensible y cosa como posibilidad de su aparecer efectivo en la realidad. Se concluirá que hay un ajuste a priori entre las cualidades y las propiedades cósicas que permitirá comprender la función presentativa del trazo sensible de las cosas.

Palabras clave: cualidades; espacio; percepción; sensación; fenomenología temprana

\begin{abstract}
The Thing which Traces Itself: Limits and Apories of Sensible Appearing from Early Phenomenology". This paper aims to investigate the presence of sensible things in the light of the works of early phenomenology. For this purpose, a reflection will be made from the problems that some thinkers of Gotinga circle outlined in the context of Husserl's theses about Abschattung. Thus, we will distinguish between things and their sensible stroke, detecting the possibilities of their mutual phenomenal connection and detachment. By this way, a triple relationship will be identified in which the empirical thing appears, composed of sensitive qualities, sensations of the perceiver subject and its spatial location, in order to examine which of these instances explains the necessary link between sensitive stroke and thing as a possibility of its actual appearance in reality. It will be concluded that there is an a priori adjustment between qualities and physical properties that will allow to understand the presentative function of the sensible stroke of things.

Keywords: qualities, space, early phenomenology, perception, sensation.
\end{abstract}

* Artículo elaborado en el marco del Proyecto Fondecyt Regular nº 1190029 
1. El aparecer abgeschattet. Las aporias del carácter (re)presentativo del trazo sensible

Logische Untersuchungen y lecciones de Husserl como Die Idee der Phänomenologie o Ding und Raum irrumpirán a inicios del Siglo XX como un singular esfuerzo del pensar. Lejos de suscribir las tendencias naturalistas y positivistas dominantes en la época, dichas obras se alzaban con objeciones profundas a la misma experiencia natural, esa para la cual las cosas ya están "dadas de manera evidente" ${ }^{1}$ y que entiende que para erigir sus conocimientos solo debe apoderarse paulatinamente de "una realidad que simplemente existe"2. Mas para dejar dicho supuesto en suspenso solo bastaba con constatar una verdad tan trivial como resulta afirmar que a las cosas cotidianas les es dado aparecer trazándose sensiblemente.

Como es sabido, Husserl caracterizó el aparecer de las cosas sensibles como Abschattung. Término usualmente traducido por "escorzos", "matiz" o "perfil"3. Ciertamente, "Abschattung" comprende tales sentidos cuando refiere al aparecer de las cosas sensibles ${ }^{4}$. Cuando estas se brindan abgeschattet, esto mienta que se presentan matizadas, bosquejadas, mas nunca plenas. El cubo, por ejemplo, sostenido en las manos deja oculto algunos de sus lados según su "esbozo perspectivístico" (perspektivistische Abschattung) 5 . Asimismo,

Husserl, E., Die Idee der Phänomenologie. Fünf Vorlesungen, La Haya: M. Nijhoff, 1958, p. 75. Ibid., p. 76.

Cf. Guíñez, P., "Husserl en el mundo hispanohablante: su traducción y recepción. Conversación con Antonio Zirión Quijano", en: Revista Pensamiento Politico, 7 (2016), pp. 255-279. También, cf. Osswald, A., "El concepto de pasividad en Edmund Husserl", en: Areté, v. XXVI, 1 (2014), pp. 33-51; Paredes Martín, M, "Percepción y atención. Una aproximación fenomenológica”, en: Azafea, 14 (2012), pp. 79-92; Respecto de las traducciones en francés, cf. Veríssimo, D., en: Psicología USP, v. XXVII, 3 (2016), pp. 521-530.

4 Cabe señalar que "Abschattung" posee una ambigüedad. Sommer distingue entre: "el proceso por el cual algo es esbozado, así como también el resultado que resulta de este proceso" (Sommer, M., "Abschattung", en: Zeitschrift für philosophische Forschung, v. L, 1/2 (1996), p. 272). Husserl, en efecto, distinguirá entre Abschattung como "vivencia" (Erlebnis) y Abgeschattetes como caracterización de la presencia espacial (Husserl, E. Ideen zu einer reinen Phänomenologie und phänomenologischen Philosophie, en: Gesammelte Schriften, v. V, Hamburgo: F. Meiner Verlag 1992b, p. 86) o explicitará Abschattung como "la exposición" (Darstellung) y Abgeschattet como "lo expuesto" (Dargestelltes) (Husserl, E., "Die Präsentation der Wahrnehmung”, en: Philosophie der Wahrnehmung. Modelle und Reflexionen, Fráncfort d. M.: Suhrkamp, p. 215). Dado a que nuestro interés es indagar el aparecer sensible mismo, daremos énfasis a Abschattung en cuanto "abgeschattet", esto es, al carácter mismo de tal aparecer en cuanto "matizado".

5 Husserl, E., Ding und Raum. Vorlesungen 1907, La Haya: M. Nijhoff, 1973, p. 102. 
el amarillo de una esfera jamás alcanza un ideal de homogeneidad, sino que en distintos sectores este se da con mayor o menor definición ${ }^{6}$. El principal problema es aquí el del cumplimiento (Erfüllung) de la cosa, uno tal que, así Husserl: "hace presentable lo que la intención mienta, pero de modo más o menos impropio o inadecuado..."7 y que, sin embargo, permite que tengamos la experiencia de que "esto es ello mismo (das ist es selbst)" la cosa, entonces, es que, a pesar de enseñarse según su esbozo sensible más o menos pleno, esta consiga de todos modos brindarse como la misma, como si cada uno de sus trazos, siempre variables, pudiesen presentar una invariabilidad inherente a ella. Y su indagación es lo que Husserl entenderá como un estudio de las relaciones de cumplimiento (Erfüllungsverhältnisse) ${ }^{9}$, uno tal que aclare cómo es que un núcleo de identidad, "[sirviendo] como fundamento a la identificación"10, se halla siempre dispuesto a especificarse sensiblemente ${ }^{11}$.

Precisamente, es en este contexto aporético que surgieron detallados trabajos por parte de discípulos de Husserl que integraron el círculo fenomenológico en Gotinga ${ }^{12}$. Suscribiendo sus principales tesis perceptivas, estudios como los de Wilhelm Schapp, Hedwig Conrad-Martius, Heinrich Hofmann, David Katz o Herbert Leyendecker se ocuparon de las aporias de la saturación sensible. Y lo decisivo es que tales trabajos venian a constatar que la relación entre las cosas y lo que aquí llamaremos "trazo sensible", entendida como "representación", no agotaba en absoluto el problema de su unidad, sino que lo abría aún más. Ciertamente, la "representación" aquí mentada es peculiar, pues refiere ante todo al modo como una cosa se "presenta a sí misma"13. El trazo sensible, es decir, todo aquello que la cosa muestra sensiblemente, es, en efecto, "presentante" (präsentierende) de ella, mas no como mero símbolo, sino en cuanto esta se trae a persona mediante él ${ }^{14}$. Mas, siendo esto así, se presentan

\footnotetext{
6 Ibid., p. 70.

7 Husserl, E., Logische Untersuchungen, Zweiter Band, II. Teil, en: Gesammelte Schriften, v. IV, Hamburgo: F. Meiner Verlag, 1992a , p. 597.

8 Ibid.

9 Ibid., p. 623.

10 Ibid., p. 618.

11 Husserl, E., "Die Präsentation der Wahrnehmung”, p. 208.

${ }^{12}$ Wilhelm Schapp brinda un cuadro de aquella época en Gotinga, exponiendo las tesis y problemáticas husserlianas decisivas para el incipiente círculo fenomenológico, así como algunas apreciaciones personales sobre algunos discípulos que lo integraron (Cf. Schapp, W. Erinnerung an Edmund Husserl. Ein Beitrag zur Geschichte der Phänomenologie, Wiesbaden: Heymann Verlag, 1976).

${ }^{13}$ Husserl, E., Logische Untersuchungen, p. 609.

${ }^{14}$ Ciertamente, el término husserliano "Re-präsentation" utilizado en Logische Untersuchungen no puede mentar la idea de símbolo, en cuanto este implicaría aceptar las tesis inmanentistas
} 
preguntas de diversa índole para estos pensadores: ¿cómo es posible, pues, que este trazo sensible pueda fallar y mostrarnos algo que no es? Leyendecker, indagando las ilusiones perceptivas, indica que "en la percepción estimamos como real una enormidad que en absoluto puede acreditarse en esa actitud perceptiva como real"15. Desde tal advertencia se desprende, a nuestro juicio, que entre la cosa y su trazo sensible muchas veces es posible que acaezca una desvinculación que finalmente anula su efectivo aparecer. Y tal desvinculación es la que parece evidenciarse más aún en otra aporía de la función presentativa del trazo sensible. Atendiendo, pues, a los matices cromáticos de una cosa, no tardamos en advertir que el orden de estos es dificilmente identificable con el orden de la cosa misma. Así, Schapp indica: "las formas y el orden que tiene lo expuesto, son por ello incomparables con el orden y la forma de lo que expone" 16 . Y con ello lo que se testimonia es que ambos, trazo sensible y cosa, terminan por separarse. ¿Cómo, entonces, es que una cosa puede traerse a persona mediante una instancia que difiere de ella misma? ${ }^{17}$ Queda, así, de manifiesto un peculiar entre en la relación entre trazo sensible y cosa donde un eventual vínculo puede perderse o acaecer. Mas, en este contexto, no podemos tampoco olvidar al sujeto percipiente y, por lo mismo, el rol de sus sensaciones corporales debiesen también ser consideradas al momento de pensar tal vínculo. ¿Qué lugar ocupan las sensaciones en la problemática aquí en juego? Siendo viable pensar que un acceso a la cosa depende de las capacidades sensitivas de nuestro cuerpo, hay casos, empero, en los que esto es dudoso. Cuando, por ejemplo, rozo mi piel con una pluma, lo que se presenta, ante todo, es la sensación corporal, es decir, siguiendo a David Katz ${ }^{18}$, el polo subjetivo, mas no las características de la pluma, esto es, el polo objetivo implicado en el tacto.

que el mismo Husserl se propone superar. Él mismo lo explicitará a partir de sus análisis de Locke, quien, postulando la tabula rasa, establecería ilegitimamente a las ideas como cuadros representantes del mundo exterior (cf. Husserl, E., Erste Philosophie, en: Gesammelte Schriften, v. VI, Hamburgo: F. Meiner Verlag, 1992c , pp. 126-127). Para una comprensión en detalle de la superación husserliana del representacionalismo, cf. Rizo-Patrón, R., "Superación del representacionalismo e inmanentismo en la génesis de la fenomenología husserliana de la percepción", en: Areté, v. XVII, 2 (2005), pp. 183-212.

${ }_{15}^{15}$ Leyendecker, H., Zur Phänomenologie der Täuschungen, Halle: Buchdruckerei des Waisenhauses, 1913, p. 153.

${ }^{16}$ Schapp, W., Beiträge zur Phänomenologie der Wahrnehmung, p. 126.

17 Ibid., p. 116.

${ }^{18}$ Katz, D., Der Aufbau der Tastwelt, Leipzig: Verlag von J. A. Barth, 1925, p. 19. 
Así, Conrad-Martius puede interrogar si acaso en la medida en que "siento a la cosa" efectivamente estoy en relación con ella ${ }^{19}$.

A nuestro juicio, tales advertencias no pueden sino dejar en evidencia que una afirmación tal como que la cosa simplemente se presenta mediante su trazo sensible es sumamente aporética ${ }^{20}$. Y, así, subrayando sus aporias, los trabajos de la fenomenología temprana realzan la urgencia de pensar una vez más en el aparecer sensible. Por ello, nuestro interés no será tanto el de realizar una exposición detenida de estos autores, sino, ante todo, quisiéramos servirnos de sus indicaciones para pensar cómo es que a la cosa le es dado hablar de sí misma mediante su trazo sensible, en el intento de aclarar su función presentativa. Y es que, siguiendo a Husserl, las cosas parecen decirnos: "tú me aprenderás a conocer según todo eso que soy, todas mis propiedades superficiales, mis propiedades interiores sensibles, etcétera"21. Mas, ¿en qué medida acaece este peculiar hablar de sí? Este es, pues, el problema al que se abocarán estas discusiones, y sobre el cual quisiéramos esbozar alguna respuesta con la ayuda de la fenomenología temprana.

\section{La triple relación empirica y la desvinculación entre trazo sensible y cosa}

Pues bien, si la tarea es aclarar la función presentativa del trazo sensible, primero debiésemos comprender su comportamiento respecto de la cosa que, mediante él, se trae a persona. Y es que su presencia sensible exhibe conductas del todo particulares. Si me inclino de rodillas ante la superficie de una mesa, no tardo en advertir la constante deformación de su figura. Lo mismo ocurre cuando acercamos, alejamos o giramos una taza con las manos. De inmediato notamos que esta se achica o se agranda o muestra lados cada vez diferentes. La presencia sensible enseña, así, una característica variabilidad que, no obstante, en un principio no parece resultar problemática para la percepción cotidiana. Nadie podría negar, pues, que, aunque variables, son esos colores,

\footnotetext{
${ }^{19}$ Conrad-Martius, H., Die erkenntnistheoretischen Grundlagen des Positivismus, Bergzabern: Buchdruckerei H. Müller, 1920, p. 98.

20 Las problemáticas de la noción de representación como donación de sí mismo en la percepción son expuestas críticamente por Rang en una lectura inmanente a la obra temprana de Husserl. Las aporías ahí destacadas están en juego también en los estudios de la fenomenología temprana (cf. Rang, B., "Räpresentation und Selbstgegebenheit. Die Aporie der Phänomenologie der Wahrnehmung in den Frühschriften Husserls", en: Phänomenologische Forschungen, 1 (1975), pp. 105-137). También, cf. Tugenhat, E., Der Wahrheitsbegriff bei Husserl und Heidegger, Berlin: W. De Gruyter,1967, pp. 73-76.

${ }^{21}$ Husserl, E., "Die Präsentation der Wahrnehmung", p. 205.
} 
texturas, sonidos, aromas o sabores los que nos informan efectivamente sobre las cosas. El ruido intenso que se escucha en la calle avisa que en el exterior pasa un vehículo de gran tamaño. Una extensión de color y brillos muestran la suavidad de la superficie de la taza. La experiencia cotidiana parece confiar, por lo pronto, en que hay una relación directa entre las cosas y esa capa sensible según la que ellas se trazan. Ambas dimensiones, entonces bien, se hallan para ella tan intimamente ligadas que parecen hablar con la misma voz. En este sentido es posible detectar un primer comportamiento del trazo sensible con la cosa que bien podriamos caracterizar como "unívoco".

$\mathrm{Y}$, sin embargo, es la propia variabilidad del trazo sensible antes referida la que también puede llevar a que la cosa paulatinamente se pierda. $\mathrm{Si}$ escucho cada vez más distante el ruido del vehículo, este dejará de acusar su tamaño, hasta que termine por desaparecer. Si miro mi escritorio con los anteojos empañados, los colores y brillos que debiesen hablarme de las cosas que se encuentran sobre él se vuelven borrosos, y con ello es la definición de los contornos de los mismos útiles la que se difumina. En tales casos, el trazo sensible exhibe un comportamiento distinto al de la antes destacada univocidad con la cosa, en tanto que, variando su grado de intensidad, esta puede desaparecer. Dicho de otra manera, la variación de intensidad sensible lleva a la cosa misma a presentarse en una indeterminación tal que puede terminar por ocultarla.

Mas su univocidad con la cosa y su variación de intensidad no son los únicos comportamientos del trazo sensible. También se acusa otra posibilidad, y que es la que interesa atender a continuación. Esta se aprecia en determinadas condiciones perceptivas como la penumbra. Si recorremos un jardín de noche, se presentan ante nosotros bultos oscuros, que bien podemos distinguir de meras sombras. Algo hay ahí delante, afirmamos, aunque lo que tenemos ante nosotros sean meras manchas negras. Solo si la brisa los moviese, advertiríamos luego que se trataba de arbustos. Es Conrad-Martius quien caracteriza esas manchas percibidas en la penumbra como un "mero esquema" 22 . Ahora bien, al mirar desde la lejanía un conjunto de casas, nuevamente divisamos diferentes manchas de color. Mas, tales colores, los mismos que en un principio debiesen referir a casas de una ciudad lejana, como lo señala Schapp ${ }^{23}$, ahora son percibidos sin una relación directa con ellas. Lo que vemos a la distancia son

${ }^{22}$ Conrad-Martius, H., Die erkenntnistheoretischen Grundlagen des Positivismus, p 74.

${ }^{23}$ Schapp, W., Beiträge zur Phänomenologie der Wahrnehmung, p. 72. 
manchas por sí mismas. Algo análogo ocurre también bajo ciertas condiciones de iluminación. El resplandor de un metal o del vidrio, volviéndose intenso, no nos permite distinguir de qué objeto se trata. El trazo sensible, en tal caso, oculta a la cosa, de modo que nos quedamos solo con brillos y resplandores.

Así, pues, en estos casos perceptivos se advierte un peculiar comportamiento del trazo sensible, a saber, que, ya no hablándonos de la cosa, él mismo ahora puede ser percibido como el efectivo objeto de la percepción. Es Conrad-Martius la que sugiere la problemática aquí en juego al comparar el ropaje sensible de las cosas con el de un objeto imaginado. Ella advierte: "lo representado (Vorgestelltes)... aparece revestido (umkleidet) con el 'abrigo de la sensibilidad'; pero este no tiene 'verdad' y, por consiguiente, no hay ninguna fuerza en él" ${ }^{24}$. Lo imaginado, pues, poseyendo lo que a una cosa le es necesario para mostrarse, como es su revestimiento sensible, no aparece, sin embargo, sino como una pura capa vacía, que, dada por sí misma, acaba siendo una "mera apariencia" (blosser Schein)25. De manera análoga, los casos descritos enseñan que el trazo sensible de las cosas puede modificarse hasta tal punto que este mismo también puede adquirir el carácter de un mero aparecer, en la medida en que se vuelve un puro esquema sensible que se muestra por sí mismo. Así, mientras que, en su comportamiento unívoco, el trazo sensible no parecía poder desligarse de la cosa, en esta última posibilidad, este pareciera subsistir por sí solo, sin un suelo auténtico, como si flotara en el aire. Por tanto, lo decisivo ahora es que según esta posibilidad de aparecer por sí mismo, el trazo sensible parece poder adquirir una particular autonomía respecto de la cosa. Una autonomía que también es detectable en situaciones cotidianas. Cuando observamos la constante desfiguración aspectual de la mesa, sabemos que ninguna de tales desfiguraciones corresponde a su auténtica figura. Aquí, la mesa y sus distintos aspectos parecen cada uno desplegarse de manera paralela.

Es en las investigaciones de Heinrich Hofmann donde se aprecian algunas distinciones importantes a partir de este paralelismo detectado entre trazo sensible y cosa. En efecto, cuando este afirma: "las cosas en distintas distancias, bajo condiciones de luz variables..., aparecen de manera diferente a como 'en realidad' son"26, no puede sino estar subrayando el derecho incuestionable,

\footnotetext{
${ }^{24}$ Conrad-Martius, H., Zur Ontologie und Erscheinungslehre der realen Welt. Verbunden mit einer Kritik positivistischer Theorien, en: Jahrbuch für Philosophie und phänomenologische Forschung, 3 (1916), p. 419.

25 Ibid., p. 418.

${ }^{26}$ Hofmann, H., "Untersuchungen über den Empfindungsbegriff", en: Archiv für die gesamte Psychologie, 26 (1913), p. 83.
} 
avalado en la misma percepción cotidiana, para establecer una escisión radical entre lo que él denomina la "cosa visual" (Sehding) y la "cosa real" (wirkliches Ding) ${ }^{27}$. Hofmann, en efecto, separa la cosa de su aparecer sensible y es dicha separación la que le permite centrarse ahora solo en la dimensión sensible y explicitar su propia composición. Es lo que este hace distinguiendo entre la "aparición simple de la cosa" (Dingerscheinung) y la "cosa visual" (Sehding). Dos momentos que pertenecen al puro ámbito del trazo sensible y que señalan cómo este parece constituirse. Así, por poner un caso, la diversa cantidad de perspectivas que obtenemos de los diferentes lados de un cubo, siendo estos un conjunto de "apariciones simples" (Erscheinungen), pueden ser entendidas como momentos parciales que compondrían una unidad visual total ${ }^{28}$. De esta manera, lo que se obtiene es que el trazo sensible poseería una constitución propia como es la de ser una unidad perceptiva integrada por momentos perceptivos parciales. Y, siendo esto así, ya podemos notar que dicha unidad perceptiva resultante, manifestándose ella misma con su propio contenido, puede, en efecto, no corresponder a la cosa real. Lo que advertimos con Hofmann es, en suma, que el vínculo que parece tan cierto en el comportamiento univoco entre trazo sensible y cosa no parece ser ahora sino circunstancial y jamás necesario.

Desde lo anterior, entonces, nos vemos confrontados con un muy particular problema. Detectando una separación entre trazo sensible y cosa, la pregunta que de inmediato se plantea es cómo esto es posible. Y es que en la experiencia cotidiana ocurre algo sumamente llamativo como es que, pese a que en ella esperamos que trazo sensible y cosa real se comporten unívocamente, y confiamos en que hay un vínculo entre ambos para tratar con las cosas diariamente, también es dicha experiencia la que advierte sobre una eventual desvinculación entre ambos. ¿Dónde, pues, podemos hallar al responsable de esta peculiar desvinculación? La respuesta no es sencilla. Por lo pronto, podríamos sentirnos inclinados a pensar que tal responsabilidad no recae sino en el trazo sensible mismo. No tardamos en advertir, pues, que este posee un propio contenido sensible como son texturas, colores, brillos o sombras. Y lo llamativo es que estos, poseyendo su propio contenido cualitativo, enseñan que para ellos no es necesario un vínculo con la cosa. El azul de la superficie de la taza o la melodía que escucho del violín, puedo siempre representármelos por sí mismos, sin que para ello deba pensar también en la taza o en el violín.

27 Ibid., pp. 80-83.

28 Ibid., p. 84. 
En la propia naturaleza del trazo sensible podriamos detectar, entonces, una eventual indiferencia respecto de un vínculo con la cosa que bien pareciera ser responsable de su desvinculación. Y, no obstante, pese a jugar un rol en ella, tal indiferencia no parece ser decisiva para que tal desvinculación acaezca. Siendo indiferente a su vínculo, el contenido sensible, en efecto, no podría sino jugar un rol pasivo, en la medida en que parece estar ahí dispuesto a que otra instancia lo vincule o no con algo. ¿Cuál es, pues, esta otra instancia?

Quizás debiésemos pensar que la responsabilidad de la eventual desvinculación entre trazo sensible y cosa recae más bien en el mismo sujeto percipiente. En efecto, sabemos que nuestros ojos no están capacitados para ver optimamente en la penumbra, por lo cual todo lo que intentemos ver en ella ha de ocultarse. Asimismo, si toco la superficie de mi mesa con la punta de mis dedos percibo ciertos accidentes de la madera que no son advertibles cuando deslizo mi antebrazo sobre ella. En este último caso, esta parece suave, quizás tanto como el vidrio, y es que los dedos son capaces de percibir mayores detalles que otras partes del cuerpo ${ }^{29}$. Así, sería factible afirmar que nuestra constitución orgánica ha de jugar también un rol en la eventual separación entre trazo sensible y cosa, pues en la medida en que se transgredan los limites de nuestras capacidades sensitivas, la cosa estaría destinada a desaparecer. Y, sin embargo, su intervención tampoco podría ser considerada como decisiva en la desvinculación aquí en juego. Y es que cuando nuestra constitución orgánica se encuentra en condiciones favorables, sí rinde lo que le está permitido. Nuestros órganos pueden, en efecto, mostrarnos mejor o peor algo, es cierto, pero esto no es responsabilidad solo de sus capacidades, pues ellas pueden enseñar lo que cabe dentro de sus límites solo si entra en juego otra instancia: la ubicación espacial. En efecto, es posible que a cierta distancia o en la penumbra no pueda distinguir eso que me sale al paso en cuanto mis ojos pierden su sensibilidad. Pero si me acerco a esas manchas negras, sí puedo reconocer luego el arbusto en mi jardín.

Así, advertimos que el contexto en el que acaece la desvinculación del trazo sensible con la cosa es un fenómeno que implica necesariamente una relación triple que en lo que sigue será decisivo tener en cuenta. Se trata de una relación en la cual aparecen las cosas empíricamente y que está integrada, como se advierte, por tres instancias, a saber, por los contenidos sensibles,

${ }^{29}$ Katz, D., Der Aufbau der Tastwelt, p. 45. 
la organización sensitiva del cuerpo ${ }^{30}$ y la ubicación espacial del sujeto percipiente. Y teniendo ahora estas tres instancias en cuenta, notamos que tanto la indiferencia de los contenidos sensibles a un eventual vínculo con la cosa, como la misma capacidad sensitiva del percipiente, no se involucran aquí sino pasivamente. $Y$ es que es la propia ubicación espacial la que parece conjugar a ambos de manera activa, en la medida, en efecto, en que es ella la que posibilita que, situados aquí o allá, el trazo sensible pueda disminuir su intensidad hasta que la cosa se pierda o que llegue a mostrarse solo por sí mismo, ocultándola. Y es la misma ubicación espacial, por su parte, la responsable también de que los órganos puedan quedar expuestos a situaciones en las que sus capacidades se vean sobreexigidas. Entonces bien, podemos advertir que, en esta triple relación, la ubicación espacial es efectivamente la responsable activa de la desvinculación entre trazo sensible y cosa. Por ello, en lo que refiere a la dimensión visual, Conrad-Martius subraya que al indagar el aparecer sensible en términos perceptivos se vuelve necesario establecer una relación de dependencia entre la aparición de las cosas y un "lugar de observación" (Sehort) ${ }^{31}$. Ahora bien, este rol de la ubicación espacial no se da solo en lo visual. Notamos también que en la cualidad misma del ruido no está implicado el tamaño del vehículo que puedo escuchar, sino que tal tamaño depende siempre de la distancia desde la que lo oigo. Y es que la diferencia del tamaño que se da entre un objeto cercano o lejano, o las variaciones en su figura, según la perspectiva, requieren para acaecer de nuestra localización, pues, según cuan lejos o cerca, o cuan inconvenientemente situados nos hallemos ante algo, la pérdida de la cosa puede resultar parcial o total. En definitiva, la desvinculación del trazo sensible es una posibilidad que solo puede ocurrir en el contexto del ámbito empírico que implica la relación entre un percipiente situado espacialmente y la cosa, lo cual, pues, da cuenta de que esta peculiar desvinculación se caracteriza por estar siempre expuesta a la contingencia de la localización perceptiva ${ }^{32}$.

Y, sin embargo, siendo este un resultado importante, solo es un paso introductorio para aclarar la función presentativa del trazo sensible. Lo que ahora urge es comprender la posibilidad de su vínculo. ¿Cómo es que este emerge? ¿Es también la ubicación espacial responsable del mismo? Si esta lo fuera, debiésemos advertir, sin embargo, que toda percepción adecuada de algo sería un fenómeno dependiente únicamente de la contingente localización

${ }^{30}$ Conrad-Martius, H., Die erkenntnistheoretischen Grundlagen des Positivismus, p. 110.

31 Ibid., p. 71.

32 Ibid., p. 93-95. 
del sujeto. Y, sin embargo, esto no se corresponde con lo que nos enseña la misma percepción cotidiana, a saber, que podemos confiar en cada caso en la univocidad entre trazo sensible y cosa para tratar con nuestro entorno. Digámoslo, entonces, así: mientras la ubicación espacial da cuenta de su desvinculación, es la vinculación entre ambos el auténtico enigma. Por ello, lo que ahora se requiere es indagar cómo es posible este vínculo. Ahora bien, son las discusiones aquí expuestas las que precisamente señalan la vía para llegar a una respuesta. Lo que ha estado en discusión es una relación triple entre las cualidades sensibles, la constitución orgánica del percipiente y su ubicación espacial, en la cual la cosa aparece. De la última, ya hemos advertido su rol activo en la desvinculación. Mas, desde ella, el eventual vínculo quedaría reducido a la mera contingencia de nuestra localización. ¿Cabría, por tanto, buscar una respuesta indagando las otras dos instancias en juego? Mas, en tanto que las cualidades sensibles acusaban una cierta indiferencia con un eventual vínculo, tampoco parecen ser ellas las que puedan brindar la solución. ¿No sería, entonces, la sensación corporal la que garantiza aquel vínculo estable entre la cosa y su trazo sensible? Pues bien, si la tarea es entender cómo el trazo sensible es presentante de la cosa, debiésemos examinar si es la sensación corporal aquel momento que permite su comportamiento unívoco, posibilitando, pues, su función presentativa.

\section{La sensación o la dudosa veracidad del máximo de cercanía corporal}

Decíamos que, pese a que la ubicación espacial sea la responsable de la desvinculación entre la cosa y su trazo sensible, estando en esta relación comprometida también la capacidad sensitiva del cuerpo, quizás podíamos hallar en ella un eventual responsable del vínculo entre ambos. Esto parece factible si pensamos que cuando nuestros ojos son afectados por colores o brillos, confiamos en que lo que ellos sienten se corresponde con rasgos ciertos de la misma cosa. Así, según los oídos sean afectados por sonidos, dicho sentir sería un índice seguro de la existencia de lo oído. O cuando queremos verificar la cualidad de algo, posamos el dedo o la mano sobre aquello para cerciorarnos

de que lo que sospechábamos es efectivo. La sensación parece, entonces, ser garante del vinculo entre trazo sensible y cosa, en cuanto, acaeciendo en mi cuerpo, confio en lo que ella me brinda.

No obstante, dicha confianza no solo implica la pura sensación corporal. Siempre es posible sentir algo que no tenga un referente en la realidad. Hemos 
de advertir, así, que su confiabilidad solo puede volverse una certeza cuando esta ha resultado de un efectivo contacto físico con las cosas. Cuando veo la superficie azul de la taza, por ejemplo, entiendo que es su condición física la que afecta mi cuerpo, el cual, a su vez, es otra instancia física, cuya peculiaridad radica en albergar las sensaciones resultantes de tal contacto. Por ello, la percepción de un objeto solo podría ser considerada cierta en la medida en que aquellas sensaciones singulares resulten de contactos efectivos con mis ojos, oídos o manos. Esta es, pues, una de las tesis centrales defendida por todo empirismo, y es una idea, por lo demás, que también parece haber estado supuesta en nuestras discusiones. Con Hofmann, en efecto, aprendiamos que la unidad perceptiva se construía de un conjunto de apariciones parciales. Ahora, pues, podemos identificar tales percepciones parciales con las múltiples sensaciones veraces resultantes del contacto corporal, para sostener que estas, reunidas, configurarian la unidad perceptiva cierta con la que tratamos. Y, por esta vía, podríamos pensar que la ubicación espacial, responsable de la desvinculación entre la cosa y su trazo sensible, podría ser ahora superada por aquellas sensaciones ciertas. Acaeciendo, pues, un contacto físico, podríamos decir, las sensaciones corporales podrian ser estimadas como aquel suelo seguro para una experiencia efectiva de las cosas, en cuanto se confirma que lo sentido no es una mera ilusión que flota en el aire. Por tanto, dado tal contacto físico, trazo sensible y cosa real ya no se desplegarian paralela sino unívocamente, derrotando la contingencia de la ubicación espacial.

Mas, siendo esta una explicación viable, no está exenta de dificultades. $\mathrm{Si}$ atendemos a dichas sensaciones parciales, avaladas en el contacto corporal, podriamos preguntar, en efecto, si todas ellas hablan de la cosa. Y es que no todo lo sentido coincide con la cosa misma que reconocemos en frente nuestro. Al observar una taza, no solo se obtienen sensaciones que se corresponden con sus rasgos propios. También tengo la sensación de brillos o sombras que nada tienen que ver con ella. Así, hay sensaciones que permanecen como un resto que excede lo que debiésemos sentir como propio de la cosa. Pero, por otro lado, cuando toco un punto de la superficie, lo que siento es, en efecto, dicho punto. Y aunque moviese mi dedo sobre ella, lo sentido seguiría siendo una sucesión de puntos táctiles. Y, pese a ello, sé que lo así sentido es una parte, un momento restringido, de algo más amplio: la taza. Por tanto, ya podemos notar que una relación entre el conjunto de las diferentes sensaciones y lo que le es propio a la cosa misma no es unívoca. No hay entre ambas una correspondencia estricta parte a parte. El conjunto de lo sentido, excediendo o restringiéndose respecto 
de lo que es propio de la cosa, parece poseer un contenido independiente de ella. Por tanto, lo que aquí se vuelve cuestionable es esa estricta identidad que pudiéramos suponer entre todo lo sentido y todo lo que pertenece a algo.

Sin embargo, podriamos insistir en que sí hay sensaciones veraces entre todo lo que puedo sentir, por lo cual la eventual excedencia o restricción antes referida no invalidaría del todo su garantía respecto de un efectivo acceso a la cosa. Solo se trataría de decidir ahora cuáles de todas estas sensaciones se acreditarian en un efectivo contacto corporal para estimar qué podemos o no conocer de ella. Y es que cuando, por ejemplo, posamos la mano sobre algo para verificar que está delante nuestro, es efectivamente ese contacto táctil el que garantiza que eso está ahí. En dicho contacto se aprecia, en efecto, un acceso definitivamente avalado por un máximo de cercanía entre la cosa y mi cuerpo. ¿Cómo dudar, pues, que tal contacto corporal es garante de un trato con ella? ${ }^{33}$ Empero, lo que debe ser aquí examinado es esa peculiar certeza que sugiere dicho máximo de cercanía corporal.

Por lo pronto, ha de advertirse que, al abordar la posibilidad de un acceso a la cosa en términos de un máximo de cercanía corporal, lo que debiéramos suponer es que hay una simetría estricta entre cercanía y un acceso adecuado, y lejanía y un acceso inadecuado a la cosa. Empero, tal simetría no es estricta. Sabemos que los sentidos pueden clasificarse en sentidos de distancia, como vista, oído y olfato, y de cercanía, como tacto y gusto ${ }^{34}$. Tomando, así, un máximo de cercanía corporal como criterio seguro para un acceso a la cosa, debiésemos suponer que serían los sentidos de cercanía aquellos más confiables al momento de tratar con ella. No obstante, el sabor, implicando un contacto del bocado con mi cuerpo, no dice nada de la cosa. Mediante él, en efecto, no advertimos su tamaño, figura u otras de sus características, sino que se presenta más bien como una cualidad tenida por sí misma, careciendo de una concordancia estricta con los aspectos del bocado que probamos. Entonces bien, el gusto, siendo un sentido de cercanía, no brinda propiedades de la cosa misma como sería esperable de un sentido que acaece en un máximo de cercanía corporal, mostrando, así, que un contacto corporal no es decisivo para un acceso a la cosa.

Y, no obstante, recurriendo ahora a lo táctil podríamos insistir en que tal máximo de cercanía corporal sí puede poseer validez en un acceso a la cosa. Es lo que ocurre con una propiedad como la dureza. Sabemos que esta

\footnotetext{
${ }^{33}$ Conrad-Martius, H., Zur Ontologie und Erscheinungslehre der realen Welt. Verbunden mit einer Kritik positivistischer Theorien, p. 423.

${ }^{34}$ Katz, D., Der Aufbau der Tastwelt, pp. 17-18.
} 
exige de un contacto corporal para darse, pues tal contacto es una condición necesaria del tacto. ¿Hallamos, pues, en la dureza una sensación que estaría estrictamente vinculada a la cosa y a mi cuerpo? Y, si esto es así, ¿podemos, entonces, corroborar que el contacto corporal sí es garante de algún acceso a la cosa? Atendamos a un caso de Conrad-Martius. Con ella podemos preguntar: ¿sentimos, en efecto, dureza cuando nos desplazamos por el piso? La pregunta apunta a la problemática central, y da a entender una cuestionable identificación entre sensación y propiedad cósica. Un examen atento no tarda en mostrar que lo sentido es algo muy distinto de la propiedad de algo. En efecto, lo que sentimos cuando caminamos es, en estricto rigor, la presión en los pies. Asimismo, cuando pongo mi dedo en la superficie de algún objeto tampoco siento la superficie misma, sino nuevamente la presión en el dedo ${ }^{35}$. Y lo que aquí se advierte es que, pese a que dureza o superficie parezcan surgir de un máximo de cercanía corporal, como el tacto lo sugiere, es, sin embargo, clara la diferencia entre lo dado como sensación en mi cuerpo y aquello que reconocemos como propiedad de la $\operatorname{cosa}^{36}$. Y es que el material de la sensación (Empfindungsmaterial) refiere estrictamente al cuerpo y no posee la forma y estructura de las cosas exteriores ${ }^{37}$. He ahí, pues, que pueda ocurrir un solo estímulo en el dedo, empero, que podamos distinguir aspectos cualitativos tan variados como suavidad, rugosidad, etcétera ${ }^{38}$. Por ello, aunque un máximo de cercanía corporal brinde un contenido, este, no obstante, es algo radicalmente distinto de los aspectos cósicos.

Ahora bien, dicho máximo de cercanía tampoco es un rasgo exclusivo de un sentido de cercanía como el tacto, sino que también se aprecia en los sentidos de distancia. En el ámbito auditivo se le advierte cuando oímos un ruido estridente, por ejemplo. Ingresando a una habitación en la que suena la música extremadamente alta, lo que sentimos no es sino la estridencia del ruido. $\mathrm{Y}$ en tal caso, lo que podía ser una melodía se vuelve ahora una pura sensación desagradable en los oídos. En el ámbito visual ocurre algo análogo, como cuando el resplandor de un objeto metálico nos encandila y quedamos con los ojos doloridos. En tales casos, dicho máximo de cercanía implica, pues,

\footnotetext{
${ }^{35}$ Conrad-Martius, H., Zur Ontologie und Erscheinungslehre der realen Welt. Verbunden mit einer Kritik positivistischer Theorien, p. 403; y Die erkenntnistheoretischen Grundlagen des Positivismus, 1920, p. 116.

${ }^{36}$ Conrad-Martius, H., Zur Ontologie und Erscheinungslehre der realen Welt. Verbunden mit einer Kritik positivistischer Theorien, pp. 423 y 428.

${ }^{37}$ Conrad-Martius, H., Die erkenntnistheoretischen Grundlagen des Positivismus, 1920, p. 123.

${ }^{38}$ Katz, D., Der Aufbau der Tastwelt, p. 24.
} 
una primacía de la pura sensación corporal, esto es, un sentir de nuestro cuerpo que termina por alejarnos de la cosa. Por tanto, un máximo de cercanía corporal, siendo detectable también en los sentidos de distancia, no solo vuelve cuestionable la eventual simetría que debiese haber entre sentidos de cercanía y condiciones favorables, y entre sentidos de distancia y condiciones desfavorables respecto de un acceso a la cosa. Lo que se advierte, ante todo, es que cuando este acaece, lo que ocurre es que en ambas clases de sentidos la relación misma con la cosa se anula. Tal anulación radica en que lo que podía mostrarse como una propiedad de algo exterior, desaparece para dejarnos ensimismados en la pura sensación. Así, casos como la presión, la estridencia del ruido o el resplandor encandilante, nos dejan de cara al ámbito de las sensaciones puras. Y lo que ellas muestran es que, resultando de un máximo de cercanía corporal, la cosa es más bien tragada por ella. Entonces, las sensaciones no pueden ser garantes de un acceso a la cosa. Intentar detectar en ellas el vínculo entre la cosa y su trazo sensible es una tarea errada, en la medida en que lo que en estas ocurre es ante todo la pérdida de dicho vínculo.

Y, no obstante, aún podría caber una última posibilidad de insistir en la veracidad de las sensaciones. Pese a todo, sigue siendo innegable el hecho de que las cosas sí se muestran en la medida en que la capacidad sensitiva de nuestro cuerpo lo permite, es decir, que sí hay una necesaria relación entre su aparecer y nuestra organización sensitiva. ¿No es, pues, tal relación la que exige insistir en su veracidad? Empero, lo que aquí debe advertirse es que dicha relación puede ser entendida en dos sentidos muy distintos. Por un lado, podríamos entender que el aparecer de las cosas es relativo a nuestros sentidos según una dependencia empíricay, por otro, podríamos entender dicha relación como una efectiva dependencia ontológica. Mas, siendo esto así, no tardamos en advertir que los casos perceptivos aquí discutidos han referido precisamente al primer tipo de dependencia: la empírica. Lo que ha estado en discusión, en efecto, son ante todo las variaciones aspectuales y la eventual desaparición sensible de la cosa, lo cual, empero, nada decide sobre su existencia. Cuando las cosas no aparecen perceptivamente, sabemos que ellas no se han vuelto una pura nada. Su existencia no depende de la capacidad sensitiva del cuerpo. Por otra parte, una auténtica dependencia ontológica es la que enseña la sensación referida al cuerpo. Pues, pese a que el dolor sea crónico, entendemos que su existencia sí depende del mismo. Por el contrario, el dulzor del azúcar o el sonido que emerge de una cuerda de violín nos hablan de su autonomía. Si mi cuerpo los dejara de sentir, ellos seguirian ahí, dispuestos a ser percibidos. Por ello, confundir 
la dependencia empírica del aparecer de la cosa con la dependencia ontológica del aparecer de las sensaciones corporales es un paso en falso. Y es que, si la relación de las cosas con el sujeto percipiente implicase una efectiva dependencia ontológica, estas, las cosas del mundo, no podrían ser entendidas, siguiendo a Conrad-Martius, sino como el producto de un mero entramado de sensaciones, cuya existencia dependería únicamente de las leyes psicológicas del sujeto ${ }^{39}$. Empero, es la experiencia cotidiana la que nos enseña que cuando una cosa deja de ser percibida, persiste en nosotros la peculiar certeza de que esta sigue $a i^{40}$. La propia experiencia cotidiana advierte, en efecto, que las cosas son algo más que un "mero telón de sensaciones corporales"41. Su diferencia, como se advierte, radica en sus respectivas naturalezas. Presión en el pie o en el dedo y dureza o superficie se distinguen porque en las últimas hay un "más"42. Y es precisamente dicho "más" el que se pierde en la pura sensación. Como decíamos, lo que antes podía ser una melodía, se vuelve ahora una pura incomodidad en los oídos. Lo que podía ser el brillo de algo, de pronto se torna una sensación desagradable en los ojos. En suma, la pura sensación, más que implicar una ganancia respecto de un acceso a las cosas, es una efectiva pérdida de ellas. La posibilidad de su identificación, como señala Katz ${ }^{43}$, no es sino artificial. Pero, ¿qué es, entonces, aquello que podemos identificar como aquel "más" que se pierde en la sensación? Veamos esto a continuación.

\section{El en sí del contenido cualitativo: la autonomía ontológica de las cualida- des sensibles}

Pues bien, decíamos que el trazo sensible, separado de la cosa, podía ser entendido como una unidad perceptiva total compuesta de apariciones simples. Advirtiendo que tal unidad no se corresponde necesariamente con la cosa real, buscábamos en aquella relación triple, donde la cosa aparece empíricamente, la posibilidad de su eventual vínculo. Implicando esta relación a la ubicación espacial, las sensaciones corporales y las cualidades sensibles, identificábamos la posibilidad de su desvinculación en la primera. Mas, en el intento de comprender su vínculo, creíamos poder identificar esas apariciones

${ }^{39}$ Conrad-Martius, H., Die erkenntnistheoretischen Grundlagen des Positivismus, p. 110.

40 Ibid., p. 61

41 Conrad-Martius, H., Zur Ontologie und Erscheinungslehre der realen Welt. Verbunden mit einer Kritik positivistischer Theorien, pp. 408-409.

${ }^{42}$ Conrad-Martius, H., Realontologie, en: Jahrbuch für Philosophie und phänomenologische Forschung, 6 (1923), p. 161.

${ }^{43}$ Katz, D., Der Aufbau der Tastwelt, p. 55. 
simples que componen la unidad perceptiva con las sensaciones corporales. Acreditadas en un contacto corporal, decíamos, la unidad perceptiva podía ser entendida como una construcción de sensaciones veraces, garantizadas por un máximo de cercanía corporal. Mas, en la medida en que este máximo de cercanía corporal más bien implicaba una pérdida de la cosa, se volvía inviable entender que son estas las responsables del vínculo entre trazo sensible y cosa. Por tanto, no siendo hallable dicho vínculo ni en la ubicación espacial ni en las sensaciones corporales, las discusiones exigen ahora permanecer en un examen del mismo trazo sensible, lo cual, por cierto, no deja de ser problemático, en cuanto detectábamos en su contenido cualitativo una indiferencia con la cosa.

Mas, ¿en qué sentido debemos entender tal indiferencia? Por lo pronto, esta es innegable. Diariamente nos confrontamos con ella y es, de hecho, la fuente de un sinnúmero de ilusiones perceptivas cotidianas. Creyendo tomar, un mechero, si ponemos un caso, al mirar de reojo el color del objeto delante nuestro nos percatamos luego que dicho color pertenecía más bien a una goma de borrar. Es Leyendecker quien entiende este "pasar por alto lo mirado" (übersehen) como la razón de un gran número de ilusiones ${ }^{44}$. Tales casos son los que llevan a pensar que el trazo sensible posee una cierta autonomía respecto de la cosa, en la medida en que parece subsistir por sí solo. Ambos, en efecto, parecen poder desplegarse paralelamente. Y, sin embargo, es importante subrayar que tal autonomía no siempre acaece en un mismo sentido.

En efecto, cuando los lados del cubo se tornan romos según una perspectiva, advertimos que su aspecto sensible y el cubo real parecen ser dos instancias separadas. Mas, en tales casos, la autonomía entre ambos depende de la contingencia de la localización, por lo que podemos caracterizarla como una autonomía empírica. No obstante, dicha autonomía no es la misma que se acusa en los brillos y sombras que observamos en algo. En tal caso percibimos determinadas cualidades, brillos y sombras, pero estas son dadas para descartarlas, como si se tratara de residuos que han de quedar de lado para tratar con eso. Se trata aquí de un tipo de autonomía distinta a la empírica, pues de esta última esperamos que el trazo sensible hable de la cosa real, aunque no lo haga, mientras que, en este último caso, a los brillos y sombras, es decir, a la cualidad sensible, mostrándose, no le compete decir nada de la cosa. Hallamos, asi, un segundo tipo de autonomía que podemos llamar residual. Y, sin embargo, hay otro tipo de autonomía y es el que se advierte ahora en el contenido mismo

${ }^{44}$ Leyendecker, H., Zur Phänomenologie der Täuschungen, p. 44.

ARETÉ Revista de Filosofía, v. XXXIII, 2, 2021 / e-ISSN 2223-3741 
de las cualidades sensibles. Es lo que ocurre con el sonido de una campana. Aquí, el tono se hace presente, pero su aparecer no habla de la campana y, sin embargo, no es algo que se deja de lado como residuo. A diferencia de brillos y sombras, el sonido exige quedarnos con él. Este, pues, enseña una peculiar desvinculación con la cosa que ya no depende de nuestra localización, ni es residual, sino que pertenece a la propia dignidad del contenido tonal. Es lo que se evidencia también al advertir que hay "cosas" que aparecen solo mediante una cualidad sensible, como el sabor o el olor ${ }^{45}$, o como el arcoíris, cuyo ser, en efecto, consiste solo en el color. En tales casos, la cualidad sensible es a la vez el propio material de lo que aparece ${ }^{46}$. Así, pues, llamaremos a esta autonomía, en cuanto refiere a la dignidad propia del contenido sensible, una autonomía ontológica.

Y tal autonomía ontológica es la que se vuelve relevante para nuestras discusiones. Ella señala, en efecto, que al trazo sensible parece pertenecerle un peculiar carácter en sí que, a su vez, vuelve comprensible tanto su autonomía empírica como residual. Y es que si las cualidades sensibles no tuviesen un contenido propio que se enseñe en sí mismo, sería imposible que el trazo sensible pueda aparecer como un mero esquema, como ocurre en la penumbra. Asimismo, si el contenido luminoso de los brillos no tuvieran ellos mismos una dignidad cualitativa propia, no podrian aparecer por sí mismos como si merodearan a las cosas. En dicho en sí hallamos, por tanto, un rasgo fundamental de las cualidades sensibles, el cual, empero, es el que vuelve sumamente dificil comprender cómo pueden llegar a presentar a las cosas. ¿Cómo podría la cosa mostrarse mediante sus cualidades, cuando, poseyendo estas un contenido propio en sí, parecieran ser instancias completamente distintas de eso que debiesen presentar?

Se ha de notar, además, que es tal carácter en sí el que hace evidente la esencial diferencia que hay entre el comportamiento del propio trazo sensible y de las cosas en la misma realidad. Es lo que se advierte en un caso tal como cuando pensamos cómo es que una cosa y una cualidad sensible se muestran nítidamente. Cuando observamos una casa desde la lejanía, no vemos sino una figura difusa. Mas, al acercarnos, su nitidez se traduce, siguiendo a Schapp, en la obtención de una figura tridimensionalmente articulada ${ }^{47}$. La nitidez de

\footnotetext{
${ }^{45}$ Conrad-Martius, H., Die erkenntnistheoretischen Grundlagen des Positivismus, p. 44.

${ }^{46}$ Conrad-Martius, H., Zur Ontologie und Erscheinungslehre der realen Welt. Verbunden mit einer Kritik positivistischer Theorien, p. 465.

47 Schapp, W., Beiträge zur Phänomenologie der Wahrnehmung, p. 75.
} 
las cosas, en efecto, radica en la definición de sus lados y figura. En cambio, la nitidez de las cualidades no se comporta así. No hay tridimensionalidad alguna que vaya definiéndose en ellas. Cuando un aroma, un color o una textura se vuelven nítidos, lo que más bien ocurre es que estos se intensifican. En efecto, el aparecer del trazo sensible depende, ante todo, de lo que con Schapp podríamos entender como un "fortalecimiento de la intensidad" (Intensitätsverstärkung) ${ }^{48}, \mathrm{y}$ es que son términos como intensificación y debilitamiento los que describen con mayor precisión la menor o mayor nitidez de las cualidades sensibles. Por otra parte, es claro también que las cosas se comportan entre ellas bajo relaciones causales. De ellas decimos que se generan o se destruyen. Sin embargo, esto no es así en las cualidades sensibles. Mientras que "la cosa se cae, se destruye"49, la cualidad táctil, sonora o cromática se debilita, mas no podriamos hablar en su caso de una eventual destrucción. Estas, más bien, en virtud de su fortalecimiento de la intensidad solo aparecen o desaparecen. Así, las cualidades sensibles se hallan al margen de las legalidades causales que gobiernan a las cosas. En otras palabras, las reglas según las cuales la cualidad sensible se comporta en la realidad, a diferencia de la causalidad entre las cosas, son las de la pura manifestación.

Entonces bien, dicho en sí de la pura manifestación es el que advierte que una descripción de las cualidades sensibles es una tarea del todo sui generis. Por lo pronto, distinguiéndose de las cosas, su en sí enseña que ellas tampoco dependen de la capacidad sensitiva del sujeto percipiente ${ }^{50}$. La cualidad melódica, en efecto, está ahí, aunque tape mis oídos, mientras que la sensación desagradable del estruendo sí depende de que yo me aleje de la sala. Asimismo, cuando toco una superficie, lo que tengo a disposición es precisamente un aspecto objetual de la mesa. Mas, frotando mi dedo fuertemente sobre ella, lo que se presenta es el calor y el ardor en mi propio dedo, es decir, nada propio de la mesa, sino un contenido sensitivo cuya existencia depende de mi cuerpo. Así, cuando advertíamos que las cualidades acusaban un "más" que se perdía en la pura sensación, ya podemos afirmar que este ha de referir a dicho carácter en sí que, no dependiendo en absoluto de mí, puede anularse. Es, por tanto, dicho en sí el que es tragado por la sensación, y es precisamente su anulación la que permite el paso que va desde la percepción de una cualidad a la vivencia de la pura sensación. Se trata, pues, de un "más" que indica que aquello que se

\footnotetext{
48 Ibid.

49 Ibid., p. 104.

50 Conrad-Martius, H., Die erkenntnistheoretischen Grundlagen des Positivismus, p. 116.
} 
muestra "refiere a algo más" que a mí mismo. Por tanto, a la cualidad sensible le es propio mostrarse como un ante mí que yo no soy, y esto ha de acaecer siempre según un carácter de distancia. Tal carácter de distancia es, en efecto, aquel rasgo esencial de las cualidades que hace imposible sostener que ellas pueden acaecer en un máximo de cercanía corporal ${ }^{51}$. El máximo de cercanía corporal es precisamente el rasgo de la sensación que anula la donación de una cualidad, porque este implica la ausencia de aquella distancia que requiere la cualidad sensible para enseñarse como cualidad de algo ${ }^{52}$. Y es dicha distancia la que jamás se pierde, ni siquiera en un caso límite como el tacto. Este, siguiendo a Conrad-Martius, no depende de un máximo de cercanía corporal para acceder a la dureza, sino ante todo de que la cualidad se presente en su distancia, esto es, según un ante mí, que permita ir más allá de la mera presión.

He aquí la autonomía ontológica de la cualidad sensible. Esta radica, entonces, en que todo contenido cualitativo posee el carácter de un en síque, no dependiendo del sujeto, se presenta ante él al modo de una necesaria distancia. Y esto es precisamente lo problemático cuando se trata de entender la función presentativa de las cualidades sensibles. ¿Cómo pueden, entonces, ser estas presentantes de la cosa, si lo que advertimos es que poseen una autonomía que las distingue radicalmente de ella? Lo que debemos hacer, pues, es profundizar en esta aporía, pues es su aclaración, a nuestro juicio, el paso necesario para poder entender finalmente cómo es que el trazo sensible puede comportarse unívocamente con la cosa y cómo es que esta se traza a sí misma mediante él.

5. El trazo sensible de la cosa: el ajuste de las cualidades a las propiedades cósicas

Pues bien, hasta aquí hemos delimitado rasgos relevantes del comportamiento del trazo sensible en el interés de comprender cuál es su función presentativa de las cosas. Este, decíamos, puede ocultarlas según su variación de intensidad o desplegarse paralelamente, sin decir nada de ellas, debido a la ubicación espacial del sujeto percipiente. Asimismo, su contenido cualitativo parecía poseer un "más" respecto de la pura sensación, que advertía sobre su carácter en sí. Dicha autonomía ontológica terminaba, sin embargo, por radicalizar una separación entre este y la cosa, confirmando que sí podria haber una

\footnotetext{
${ }^{51}$ Conrad-Martius, H., Zur Ontologie und Erscheinungslehre der realen Welt. Verbunden mit einer Kritik positivistischer Theorien, p. 403.

52 Ibid., pp. 450 y 509. 
natural desvinculación con ella, al modo como lo sugería Hofmann. Así, desde lo discutido, ya es importante destacar que no puede sino resultar sumamente problemático afirmar sin más que la realidad se dona sensiblemente. ¿Dónde, pues, hallar finalmente aquel vínculo entre trazo sensible y cosa que nos aclare su función presentativa? ¿Cómo, pues, entender este vínculo?

Quizás sea necesario volver a la relación entre las apariciones simples parciales y la unidad perceptiva total. Con Hofmann advertiamos que la última se compone de las primeras. $\mathrm{Y}$, sin embargo, este advierte que, pese a que muchas veces este sea el caso, no siempre es así: "la 'simple aparición' [-dice él-] puede representar (repräsentieren) perceptivamente la cosa misma, y una posible cosa visual no se constituye en absoluto"53. Schapp, por su parte, señala que en ocasiones solo basta una aparición simple para tratar con algo, como cuando, tras un vistazo rápido, tomamos un lápiz, sin que requiramos percibir mayores detalles de é $1^{54}$. Así, lo decisivo aquí es que no solo la unidad perceptiva total, sino también las apariciones simples parciales poseen carácter presentativo. En cualquiera de ambos casos es la propia experiencia cotidiana la que parece asumir que hay un vínculo ya dado entre el trazo sensible y la cosa, aunque dicho trazo sea incompleto. Para ella, en efecto, el vínculo no es el problema. Al contrario, para ella el auténtico obstáculo es más bien la anulación del mismo. Por ello, cuando el brillo impide saber con qué hemos topado, no aceptamos sin más tal impedimento, sino que intentamos superarlo. "Según lo que se quiera ver del objeto, [-dice Schapp-] siempre se debe buscar continuamente otros lugares, hacer uso de otras apariciones parciales (Erscheinungen)" 55 . He aquí, pues, un esfuerzo que señala, a nuestro juicio, un muy particular rasgo de la experiencia cotidiana como es que esta se despliega ya en la seguridad de que las cosas han de presentarse sensiblemente, antes de que se dé una relación empírica con ellas. En efecto, en la experiencia cotidiana se alberga una característica confianza de contar con la univocidad entre trazo sensible y cosa que parece sugerir que el buscado vínculo precede al ámbito empírico. Pero, ¿dónde puede adquirir su derecho tal afirmación?

Pues bien, la autonomía ontológica de las cualidades sensibles ha mostrado que estas, dado su carácter en sí, pueden brindarse desvinculadas de las cosas, como el sonido, el sabor o el aroma. Sin embargo, ha de advertirse que algo de su vinculación se conserva. Pese a que la melodía pueda ser

\footnotetext{
${ }^{53}$ Hofmann, H., "Untersuchungen über den Empfindungsbegriff", p. 91. Cf., además, ibid., p. 87.

${ }^{54}$ Schapp, W., Beiträge zur Phänomenologie der Wahrnehmung, p. 93.

55 Ibid., p. 86.
} 
tomada por ella misma, sabemos, empero, que esta surge del instrumento ${ }^{56}$, así como nos percatamos de que tal o cual cosa es la que sabe o huele de tal o cual manera ${ }^{57}$. Tales cualidades son, empero, flexibles respecto de la cosa. Ahora bien, tal flexibilidad se advierte también en lo visual, como en los brillos. Estos, decíamos, merodean la cosa sin encontrar en ella un auténtico asidero. No obstante, resulta interesante que el mismo brillo, pudiendo ser solo un residuo, en ocasiones sí pueda ser decisivo para que algo se muestre. Es lo que ocurre con la superficie del agua, cuyo nivel, advierte Schapp, es perceptible, en efecto, mediante sus brillos y reflejos ${ }^{58}$. ¿Cómo ocurre, pues, que tales brillos, a veces, intensificándose, oculten a la cosa o que sean meros residuos y que, en otras ocasiones, sí puedan llegar a tener una efectiva función presentativa? ¿En qué radica tal modificación? Digámoslo así: en el caso del agua, su brillo presenta un peculiar rasgo que lo distingue de los otros casos. Un rasgo que también se advierte en los colores. Respecto de ellos, Schapp señala: "Cuando un ser tiene color en este orden, luego este ve objetos, cosas, y mientras este no tenga al color en este orden, no ve las cosas, sino que tiene solo, en cierta manera, color. Esta es una proposición a prior" ${ }^{\prime 59}$. Entonces, algo puede suceder con los brillos que también ocurre con los colores, y que modifica su condición de meros residuos, a saber, que ellos pueden darse no solo en sí mismos sino, a la vez, como vinculados al orden de la cosa. En efecto, aunque los brillos puedan, a veces, no ser tomados como cualidades cósicas, en los casos en que esto sî ocurre, es porque su aparecer se brinda ajustado precisamente a dicho orden.

He aquí un rasgo que marca la radical diferencia entre el aparecer de la cualidad por sí misma y su aparecer como cualidad de algo. A saber: para que las cualidades dejen de ser cualidades puras, como el color o el aroma por sî mismos, y adquieran el carácter de cualidades cósicas, han de aparecer, ante todo, ajustadas a un orden cósico. La cualidad, en efecto, puede mostrar a la cosa solo en cuanto ella se brinde, en palabras de Conrad-Martius, como "formada" (geformt $)^{60}$. Es lo que se advierte en el sonido. Pese a su flexibilidad

$286{ }^{56}$ Conrad-Martius, H., Zur Ontologie und Erscheinungslehre der realen Welt. Verbunden mit einer Kritik positivistischer Theorien, p. 469.

${ }^{57}$ Hans Henning distingue la donación olfatoria pura (reine Gegebenheitsgeruch), es decir, la experiencia del olor como una pura cualidad en sí, sin referencia a algo, y el olor objetual (Gegenstandsgeruch), que indica la experiencia del olor remitida a algo (Henning, H., Der Geruch, Leipzig: J.A. Barth Verlag, 1916, p. 29).

${ }_{58}$ Schapp, W., Beiträge zur Phänomenologie der Wahrnehmung, p. 86.

59 Ibid., p. 110.

${ }^{60}$ Conrad-Martius, H., Zur Ontologie und Erscheinungslehre der realen Welt. Verbunden mit einer Kritik positivistischer Theorien, p. 499. 
respecto de la cosa, este sí puede aparecer formado como "ruido de algo". Así, aún cuando podamos decir que el ruido de la calle es un auténtico caos, lo que escuchamos sigue dándose en una tal configuración que, lejos de ser una pura sensación, se brinda como el ruido de la calle. Por tanto, sin tal carácter de formado según el orden de la cosa, las cualidades han de quedar vedadas de su función presentativa ${ }^{61}$. Y, asimismo, en casos en los que la cualidad llega a ocultar tal orden, como ocurre cuando el brillo se intensifica, ella también ha de dejar de hablar de la cosa 62 .

Entonces bien, es tal carácter "formado" aquel rasgo que debemos entender como esencial para distinguir entre el aparecer de una cualidad pura y el aparecer de una cualidad cósica. En otras palabras, para que ocurra el acceso a algo no basta solo con la cualidad pura. Cuando esta pasa a ser cualidad cósica, más allá de que su contenido cualitativo posea el carácter de en sí, debe hallarse también ajustada a la $\operatorname{cosa}^{63}$. Y es en este sentido que Schapp puede denominar a los colores con función mostrativa "colores adheridos" (anhaftete Farben) ${ }^{64}$. Tal adhesión es la que subraya precisamente aquel ajuste al orden cósico que finalmente brinda a las cualidades su función de "expositoras" (Darstellende). Entonces bien, si a partir de la autonomía ontológica de la cualidad advertíamos un esencial carácter en sí que se presenta ante el sujeto según una necesaria distancia, ahora entendemos que dicha distancia, en el caso de las cualidades cósicas, no refiere solo a dicho en sí. Cuando la cualidad pura se configura como cualidad cósica, esto ocurre siempre en virtud de un mostrarse perteneciendo a algo otro, lo cual es precisamente aquel rasgo que da paso a la experiencia de la cualidad de algo que podríamos caracterizar, con Conrad-Martius, como un "captar lo que me confronta" (Entgegennahme) ${ }^{65}$, es decir, como un tener algo al frente mío que permite precisamente dar un salto (Übersprung) a la cosa66. He ahí, pues, que esta caracterice el contenido cualitativo cósico como un "contenido testimonial" (Kundgabegehalt) ${ }^{67}$. Precisamente, la cualidad cósica es en esencia testimonio de la cosa, en la medida en que el en sí de su contenido sensible, pese a no dejar de aparecer en sí mismo, se da,

61 Ibid., p. 478.

62 Schapp, W., Beiträge zur Phänomenologie der Wahrnehmung, p. 108.

${ }^{63}$ Conrad-Martius, H., Zur Ontologie und Erscheinungslehre der realen Welt. Verbunden mit einer Kritik positivistischer Theorien, p. 467.

${ }^{64}$ Schapp, W., Beiträge zur Phänomenologie der Wahrnehmung, p. 87.

65 Conrad-Martius, H., Zur Ontologie und Erscheinungslehre der realen Welt. Verbunden mit einer Kritik positivistischer Theorien, p. 481 y 533.

${ }^{66}$ Conrad-Martius, H., Die erkenntnistheoretischen Grundlagen des Positivismus, p. 88 y 126.

67 Ibid., p. 54. 
empero, en aquella peculiar configuración como la de venir desde algo otro, es decir, como ya ajustado al orden cósico.

$\mathrm{Y}$, sin embargo, ¿cómo entender dicho orden? ¿Debemos simplemente identificarlo con la figura de algo? ¿No son, pues, las figuras las que sugieren un orden en la cosa? Mas, son ellas las que constantemente varian según las perspectivas. Las cosas continuamente se deforman según la contingente ubicación espacial del sujeto. Y, asimismo, aspectos que ellas mismas puedan sugerir, como el tamaño, también quedan expuestos a su variación. El tamaño, en efecto, se encoge o agranda según nos localicemos cerca o lejos de algo. Por tanto, si es que el trazo sensible debiese dar un efectivo testimonio de las cosas, no parece ser posible que este se encuentre en relación con las meras figuras. Empero, lo decisivo aquí es notar que, pese a lo anterior, sî hay una experiencia de la figura y del tamaño auténtico de algo ${ }^{68}$. Es lo que se advierte cuando distinguimos dos experiencias muy distintas como son, por un lado, el achicamiento de algo debido a la distancia y, por otro, el encogimiento de una cosa por sí misma ${ }^{69}$. Dicha diferencia no deja de ser relevante, pues sugiere que sí hay un orden cósico invariable. Y, a la vez, ella señala que tal orden parece no hallarse sujeto al ámbito de las relaciones empíricas que solo brindan variación aspectual. Entonces bien, ¿dónde debemos indagarlo? Y, ¿de qué manera la cualidad cósica se vincula a él?

Tomemos el impacto de dos bolas de billar. Del mismo podemos distinguir, por un lado, un nivel cósico, esto es, la bola que impulsa a la otra en una determinada dirección y, por otro, un nivel sensible, es decir, el sonido y las variaciones cromáticas ahí percibidas. Ambos niveles poseen, por cierto, unidad. El ruido enseña el choque, y el color, el desplazamiento de las bolas. No obstante, antes de ello, este ha enseñado algo aún más decisivo. Y es que, si el ruido del choque no advirtiera primero sobre el peso y dureza de ambos cuerpos, no podríamos luego comprender la fuerza del impacto o la velocidad

\footnotetext{
${ }^{68}$ Hofmann distingue del tamaño objetivo medible, aquel que es el tamaño visual (Sehgrösse), variable según un achicamiento o agrandamiento perspectivístico. Dicho tamaño es puesto en relación con el tamaño natural (natürliche Grösse), que es con el que cotidianamente contamos y que implica una cercanía con las cosas que permita aún distinguir el todo y sus partes, y una lejanía en la que aún sea distinguible tal tamaño acostumbrado ( $c f$. Hofmann, H., "Untersuchungen über den Empfindungsbegriff”, pp. 62-69). Conrad-Martius, distinguiendo el tamaño de la cosa (Dinggrösse) del tamaño aspectual de la cosa (Dinggrössenaspekt), realza la dependencia del último a la relación empírica, mientras que vincula al primero con un orden a priori que ella entenderá como la materia (Materie) (cf. Conrad-Martius, H., Die erkenntnistheoretischen Grundlagen des Positivismus, pp. 76-79).

69 Conrad-Martius, H., Die erkenntnistheoretischen Grundlagen des Positivismus, p. 79.
} 
y dirección de la trayectoria del cuerpo empujado. Son precisamente peso y dureza, es decir, esas propiedades determinadas, las que hacen comprensibles tal relación. Así, Schapp señala que los colores adheridos "muestran la consistencia de la madera, lo pulido del metal, la blandura del pañuelo. Ellos persiguen la estructura de la cosa hasta lo más íntimo"70. Y, como se advierte, son precisamente las propiedades las que aquí son entendidas como eso más intimo. Cuando oímos, pues, el ruido de un camión, oímos el peso que nos sugiere su tamaño. El trazo sensible, entonces, parece ser más específico en su función mostrativa que solo referir a la mera figura: "El color, la figura, el movimiento [-dice Schapp-] nos traen a presentación a la cosa. Pero en realidad, ellos nos traen a presentación solo propiedades de la cosa..." ${ }^{11}$. Y es que estas, las propiedades, son aquel momento primario en ellas. En efecto, Schapp entenderá análogamente a la cosa y sus propiedades con las personas y su carácter. Él señala: "cada hombre se ubica bajo un carácter, él no puede actuar de otra manera que según las propiedades de su carácter"72. Y así como las personas no pueden actuar sino según su carácter, las cosas tampoco pueden comportarse de manera distinta a sus propiedades. Cuando dos cuerpos sólidos impactan, indica Schapp, el golpe acaece como una inevitable resistencia, del mismo modo que cuando confrontamos a una persona dura de carácter. Pero cuando una bola de metal impacta la greda, ella es tragada por esta, y el choque se disuelve de la misma manera que al confrontar a una persona apacible (sanfmütig) ${ }^{73}$. Las propiedades de las cosas son, por tanto, aquello intimo en lo que ellas mismas radican. Siendo esto así, ya podemos advertir que cuando estas se muestran sensiblemente, lo hacen exhibiendo ante todo sus propiedades, pues es desde ellas que las cosas se muestran a sí mismas. Es, en definitiva, en este sentido que debemos entender que las propiedades son su más propia individualidad. Así, Schapp afirma: "La cosa solo mantiene su individualidad, no su espacio frente a las otras [cosas]. Ella convive con estas según su carácter" 74 .

He aquí, entonces, lo decisivo. Manteniéndose la cosa a sí misma según sus propiedades, esta, en lo que respecta a su propia constitución, jamás ha estado sujeta a su aparecer empírico. Precisamente es con Schapp que se advierte que las propiedades no son ellas mismas espaciales y no deben su

\footnotetext{
${ }^{0}$ Schapp, W., Beiträge zur Phänomenologie der Wahrnehmung, p. 90.

Ibid., 116.

2 Ibid., p. 119.

${ }^{73}$ Ibid.

${ }^{74}$ Ibid., p. 123.
} 
orden y existencia a su aparición empírica ${ }^{75}$. En otras palabras, configurándose en su propia individualidad desde sus propiedades, a las cosas solo les es dado manifestarse aspectualmente en lo empírico, sin que para ellas esto sea relevante. Podemos, pues, tratar solo con el blanco del azúcar, sin que su dulzor se nos brinde. Mas, este sigue ahí como propiedad, sin que su ausencia aspectual le afecte en absoluto ${ }^{76}$. Por ello, podemos afirmar que el vinculo entre trazo sensible y cosa no depende de la relación empírica, pues este ya se encuentra dado antes: en la cosa misma, esto es, en la identidad configurada por sus propiedades.

Es por ello que la relación aquí en juego ha de ser entendida como a priori respecto de su aparecer empírico, es decir, como previa a su aparición meramente aspectual. Y es que cuando una "parte" de tal todo se muestra, esta, lejos de ser un segmento de una figura inabarcable perspectivísticamente, se dona más bien como un momento determinado de la identidad de la cosa. Dicho de otro modo, cuando una propiedad se esboza sensiblemente, la cosa, en efecto, ya ha conseguido mostrarse a sí misma y, con ello, el trazo sensible ya ha cumplido su función presentativa. El ruido, por ejemplo, brinda precisamente el peso del vehículo, y he ahí que él es efectivo en su presentar, porque es dicho peso el modo como en ese momento este aparece. Mas, si ahora exigiéramos del ruido una presentación del tamaño exacto del vehículo, violentariamos su función presentativa. Así, aunque no todas las propiedades se brinden en la cosa, lo cierto es que una vez que una de ellas lo ha hecho, ya la tenemos ante nosotros, al menos en un momento de su identidad que nos garantiza su presencia. Si a veces, por tanto, solo bastan apariciones simples parciales para un trato con ella, esto se debe justamente a que su pobreza en detalles aspectuales no pretende hablar de su figura, sino más bien de alguna de sus propiedades que componen su identidad. Es en este sentido, en definitiva, que podemos sostener que la eventual relación del trazo sensible y la figura empírica de la cosa es un fenómeno más bien secundario respecto de su vínculo con las propiedades. Y, por otra parte, advirtiendo lo decisivo de esta última relación, es decir, comprendiendo el aparecer sensible de las cosas como la donación empírica de sus propiedades, se hace posible entender, según lo discutido, aquella confianza de la experiencia cotidiana en un comportamiento unívoco de la cosa y su trazo sensible.

${ }^{75}$ Ibid., p. 117.

${ }^{76}$ Cf. Conrad-Martius, H., Die erkenntnistheoretischen Grundlagen des Positivismus, p. 60. 
He ahí, pues, la peculiaridad del trazo sensible de las cosas, y he ahí que la relación entre ambos sea una verdadera aporía. Empero, llegando a este punto, creemos haber obtenido algunos resultados relevantes en el intento de comprender cómo acaece su función presentativa. A nuestro juicio, es ese particular ajuste del mismo a las propiedades cósicas la vía a seguir para indagar la aporética donación sensible. Sin embargo, estas discusiones no han pretendido cerrar los problemas, sino más bien abrirlos. Ciertamente, una investigación como la aquí propuesta exige pasar ahora a un nivel que aprehenda aquel sentido intencional según el que un mundo concreto se nos brinda en su unidad. Mas, antes de avanzar en dicha dirección, era necesario, a nuestro juicio, detenerse en las aporias implícitas en el comportamiento mutuo que exhibe la cosa y su trazo sensible, permaneciendo en un nivel inicial de consideración que indagara primero cómo es que las cualidades presentan a la cosa y nos permiten tratar efectivamente con ellas. Ahora bien, esto no habría sido posible sin la ayuda de la fenomenología temprana. Tales estudios, en efecto, despiertan aquel espíritu crítico y autónomo que hemos intentado ensayar a lo largo de este trabajo. Más allá, pues, de su interés histórico, ellos brindan la posibilidad de mantener vivo el carácter enigmático de la Abschattung que Husserl plantearía como vía para pensar la donación de las cosas, y su vigencia radica precisamente en que aún llaman a cultivar una reflexión detallada sobre aquello que el mismo Husserl interrogó, esto es: cómo es que las cosas nos hablan de sí mismas cuando se esbozan sensiblemente. Sobre esto, empero, queda mucho que decir. Este es solo un primer paso.

Recibido: 03/05/2021

Aceptado: 27/08/2021

\section{Bibliografia}

Conrad-Martius, H., "Zur Ontologie und Erscheinungslehre der realen Welt. Verbunden mit einer Kritik positivistischer Theorien", en: Jahrbuch für Philosophie und phänomenologische Forschung, 3 (1916), pp. 345-542.

Conrad-Martius, H., Die erkenntnistheoretischen Grundlagen des Positivismus, Bergzabern: Buchdruckerei H. Müller, 1920.

Conrad-Martius, H., Realontologie, en: Jahrbuch für Philosophie und phänomenologische Forschung, 6 (1923), pp. 159-333.

Grimm, J. y W. Grimm, Deutsches Wörterbuch, versión online en: http://woerterbuchnetz.de/cgi-bin/WBNetz/wbgui_py?sigle=DWB 
Guíñez, P., "Husserl en el mundo hispanohablante: su traducción y recepción. Conversación con Antonio Zirión Quijano”, en: Revista Pensamiento Político, 7 (2016), pp. 255-279.

Henning, H., Der Geruch, Leipzig: J.A. Barth Verlag, 1916.

Hofmann, H., "Untersuchungen über den Empfindungsbegriff”, en: Archiv für die gesamte Psychologie, 26 (1913), pp. 1-135.

Husserl, E., "Die Präsentation der Wahrnehmung”, en: Philosophie der Wahrnehmung. Modelle und Reflexionen, Fráncfort d. M.: Suhrkamp, 2017.

Husserl, E., Die Idee der Phänomenologie. Fünf Vorlesungen, La Haya: M. Nijhoff, 1958. Husserl, E., Ding und Raum. Vorlesungen 1907, La Haya: M. Nijhoff, 1973.

Husserl, E., Logische Untersuchungen. Zweiter Band., II. Teil, en: Gesammelte Schriften, v. IV, Hamburgo: F. Meiner Verlag, 1992a.

Husserl, E., Ideen zu einer reinen Phänomenologie und phänomenologischen Philosophie, en: Gesammelte Schriften, v. V, Hamburgo: F. Meiner Verlag, 1992b.

Husserl, E., Erste Philosophie, en: Gesammelte Schriften, v. VI, Hamburgo: F. Meiner Verlag, 1992c.

Katz, D., Der Aufbau der Tastwelt, Leipzig: Verlag von J. A. Barth, 1925.

Leyendecker, H., Zur Phänomenologie der Täuschungen, Halle: Buchdruckerei des Waisenhauses, 1913.

Osswald, A., "El concepto de pasividad en Edmund Husserl", en: Areté, v. XXVI, 1 (2014), pp. 33-51.

Paredes Martín, M., "Percepción y atención. Una aproximación fenomenológica", en: Azafea, 14 (2012), pp. 79-92.

Rang, B., "Räpresentation und Selbstgegebenheit. Die Aporie der Phänomenologie der Wahrnehmung in den Frühschriften Husserls”, en: Phänomenologische Forschungen, 1 (1975), pp. 105-137.

Rizo-Patrón, R., "Superación del representacionalismo e inmanentismo en la génesis de la fenomenología husserliana de la percepción”, en: Areté, v. XVII, 2 (2005), pp. 183-212.

Schapp, W., Beiträge zur Phänomenologie der Wahrnehmung, Fráncfort d. M.: V. Klostermann, 2013.

Schapp, W., Erinnerung an Edmund Husserl. Ein Beitrag zur Geschichte der Phänomenologie, Wiesbaden: Heymann Verlag, 1976.

Sommer, M., “Abschattung”, en: Zeitschrift für philosophische Forschung, v. L, 1 /2 (1996), pp. 271-285.

Tugendhat, E., Der Wahrheitsbegriff bei Husserl und Heidegger, Berlín: W. De Gruyter, 1967. https://doi.org/10.1515/9783111523071

Veríssimo, D., "Husserl's Theory of Perceptive Donation according to Profiles", en: Psicología USP, v. XXVII, 3 (2016), pp. 521-530. https://doi.org/ 10.1590/0103-656420150043 